\title{
Acoustic Wave Filter Based on Periodically Poled Lithium Niobate
}

\author{
Emilie Courjon, Florent Bassignot, Gwenn Ulliac, Sarah Benchabane, and Sylvain Ballandras
}

\begin{abstract}
Solutions for the development of compact RF passive transducers as an alternative to standard surface or bulk acoustic wave devices are receiving increasing interest. This article presents results on the development of an acoustic band-pass filter based on periodically poled ferroelectric domains in lithium niobate. The fabrication of periodically poled transducers (PPTs) operating in the range of 20 to $650 \mathrm{MHz}$ has been achieved on 3-in (76.2-mm) 500- $\mu$ m-thick wafers. This kind of transducer is able to excite elliptical as well as longitudinal modes, yielding phase velocities of about 3800 and $6500 \mathrm{~m} \cdot \mathrm{s}^{-1}$, respectively. A new type of acoustic band-pass filter is proposed, based on the use of PPTs instead of the SAWs excited by classical interdigital transducers. The design and the fabrication of such a filter are presented, as well as experimental measurements of its electrical response and transfer function. The feasibility of such a PPT-based filter is thereby demonstrated and the limitations of this method are discussed.
\end{abstract}

\section{INTRODUCTION}

$\mathrm{T}$ HE demand for highly coupled high-quality acoustic wave devices has generated strong innovative activity, yielding the investigation of new excitation principles [1]. Recently, we have investigated periodically poled transducers (PPTs) on single-crystal lithium niobate $\left(\mathrm{LiNbO}_{3}\right)$ Z-cut plates. A PPT consists of two electrically conductive media embracing a periodically poled ferroelectrics layer [2]. Its fabrication is performed by locally forcing the $\mathrm{LiNbO}_{3}$ ferroelectric polarization using a poling bench [3]. The poling bench mainly consists of a high-voltage amplifier used to submit the wafer to an electric field via liquid electrodes (a saturated lithium chloride solution) strong enough to override the material coercive field and, hence, to invert domains defined by an in situ photo-resist mask. PPTs then allow for the excitation of symmetrical Lamb modes [2] with operating frequencies twice as high (considering a given mechanical period) as the operating frequencies obtained using SAWs excited by standard interdigital transducers (IDTs). This principle has been successfully implemented and tested for the excitation of waves at frequencies ranging from a few megahertz [4] up to several gigahertz [5].

In this work, the fabrication of PPTs on single-crystal $\mathrm{LiNbO}_{3}$ Z-cut substrates is proposed to develop acoustic filters. For that purpose, the possibility of transposing the well-known principle of transversal SAW filters

The authors are with the Franche Comte Electronique Mecanique Thermique et Optique-Sciences et Technologies (FEMTO-ST) Institute, Besançon, France (e-mail: emilie.courjon@femto-st.fr). (based on tapered IDTs [6]) to PPTs has been considered. The interest of this approach relies on the possibility of controlling the impulse response of the transducer using plane electrodes which are easy to shape according to the intended spectral function. Because this technology is revealed to be more robust than IDT-based solutions (particularly against short circuit and lithography defects) [2], [4], [5], it should represent an attractive alternative for the development of RF filters. PPTs exhibiting a poling period of $40 \mu \mathrm{m}$ have been successfully fabricated for the experimental assessment of the proposed approach. Several designs of electrodes have been tested to fabricate a band-pass filter operating near $100 \mathrm{MHz}$. Test devices have been measured using an RF tip-probing machine. Although these devices did exhibit multi-mode responses because of the thickness of the poled $\mathrm{LiNbO}_{3}$ plate, typical filter responses have been observed and analyzed. Furthermore, acoustic-optical measurements have been performed on the corresponding devices, enabling observation of the actual distribution of the excited wave under the electrodes. These measurements enable the definition of the appropriate electrode design to fabricate an acoustic filter.

The first section of the paper is devoted to a brief review of PPTs' basic principles and specific features. Simulation fundamentals and basic fabrication methods devoted to PPTs are also summarized to introduce the filter implementation sections. Thus, the design of the filter is described first, followed by a report of the experimental characterization of the test devices, yielding discussions on the actual operation of PPT-based band-pass filters. As a conclusion, further developments of such devices are considered.

\section{Periodically Poled Transducers}

\section{A. PPTs Versus IDTs}

A PPT is based on a periodically poled piezoelectric substrate metalized on its both sides to obtain a capacitive dipole in which an elastic wave can be excited by phase construction [2]. Fig. 1 presents a comparison between classical IDTs and PPTs.

PPTs take advantage of two principal features compared with standard periodic interdigitated electrode transducers deposited atop piezoelectric materials. The first feature is the robustness of the excitation to defects or surface contamination (nondistributed electrodes with separated polarity on each side of the substrate secure the excitation and no passivation is required to avoid short- 


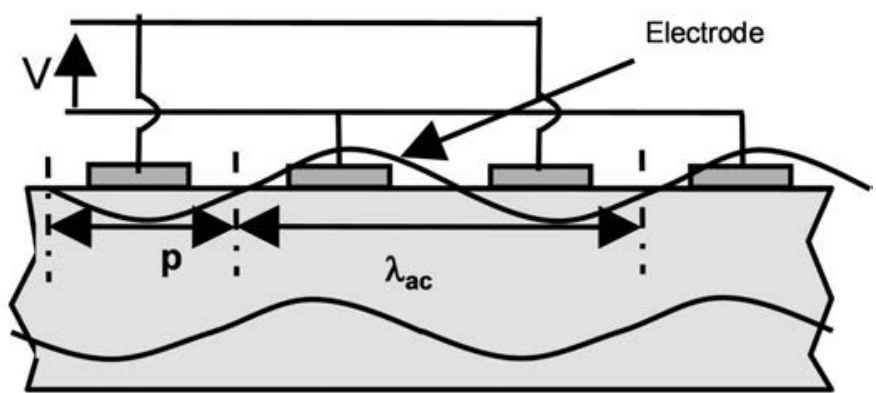

(a)

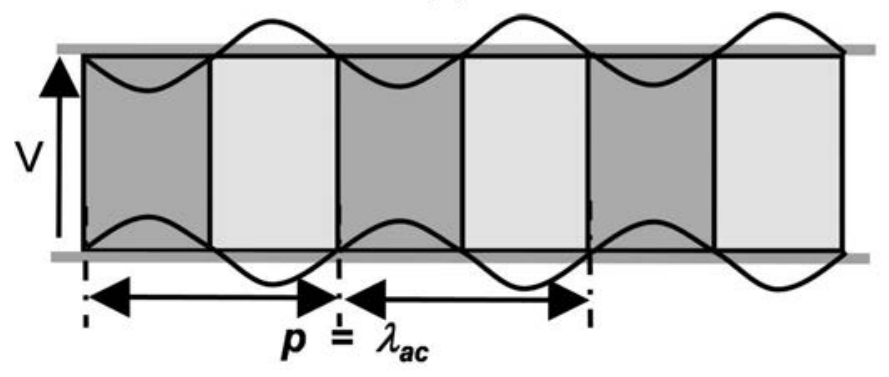

(b)

Fig. 1. (a) Classical interdigital transducer and (b) periodically poled transducer.

circuit as in the case of standard IDTs). The second feature is the possibility of exciting waves exhibiting a wavelength equal to the poling period, contrary to standard IDTs, for which the wavelength is twice the mechanical period of the grating (the Bragg condition). This characteristic, however, implies that the device operates in a second-harmonic regime, yielding the superimposition of progressive direct and backward modes. For this situation, one may expect some operational differences compared with standard IDTs. This point will be discussed in the sections devoted to filter design and manufacture.

\section{B. PPT Simulation}

A PPT can be defined by a period equal to the acoustic wavelength, a thickness, an acoustic aperture and an overall length. Because the acoustic aperture usually is much larger than the thickness of the plate and the period of the grating, a 2-D analysis can be advantageously implemented without any loss of generality. Because of the inhomogeneous nature of the material properties of the periodically poled substrate, numerical computation tools such as finite element analysis (FEA) are well adapted to predict the electrical response of PPTs. Furthermore, PPTs' structures are often periodic, with several tenths of periods along the transducer length. Therefore, meshing only one period of the transducer consisting of two rectangles representing the two opposite ferroelectric domains and considering periodic boundary conditions allows for reliable and effective computations of PPTs harmonic admittance [7]. The mesh used in our simulations is shown in Fig. 2.

As explained previously, all of this area must be meshed because of the heterogeneous piezoelectric prop-

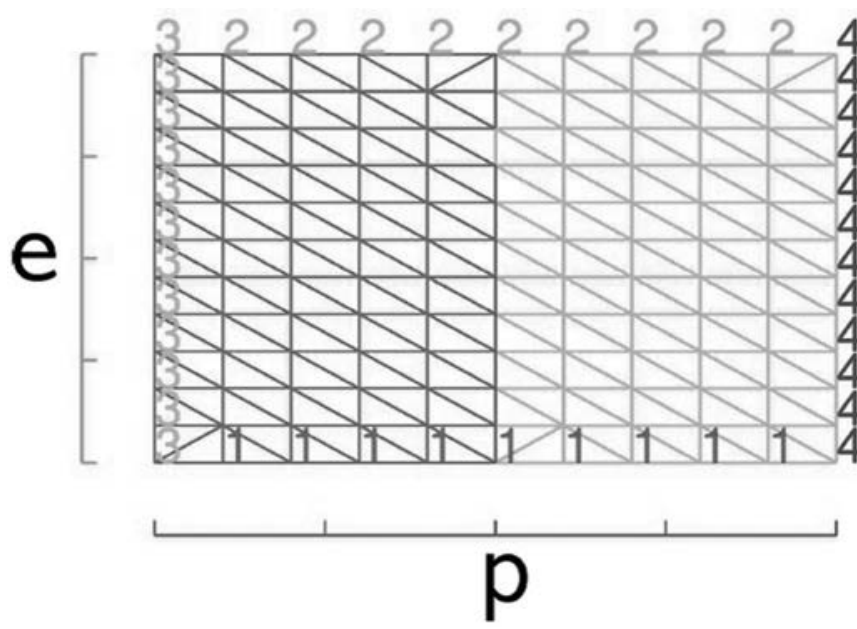

Fig. 2. Two-dimensional mesh of a periodically poled transducer.

erties of one PPT period. According to the definition in the IEEE Std-176 standard [8], the $Z+$ polarized domain corresponds to the $(\mathrm{YXl}) / 90^{\circ}$ cut whereas the second domain $\left(Z\right.$ - polarized) is a $(\mathrm{YXl}) /-90^{\circ}$ cut. The numbers on the mesh edges correspond to the references characterizing the boundary conditions applied to the mesh limits. In our case, the references labeled 1 and 2 correspond to the applied electric potential and the references labeled 3 and 4 are representative of the periodicity conditions. The simulations based on periodic FEA allow derivation of the harmonic admittance of the devices [7], which provides information about the PPT's characteristics. One can determine the nature and the synchronization frequency of the excited waves, but also the electromechanical coupling of the corresponding mode and dispersion properties, considering appropriate assumptions [9]. Fig. 3 shows the harmonic admittance of a $500-\mu$ m-thick $\mathrm{LiNbO}_{3} \mathrm{PPT}$ with a $50-\mu \mathrm{m}$ period.

Two main contributions are indicated. The first mode is excited at $76 \mathrm{MHz}$ [corresponding to a phase velocity $(V \varphi)$ equal to $\left.3800 \mathrm{~m} \cdot \mathrm{s}^{-1}\right]$ and the second mode at $131 \mathrm{MHz}\left(V \varphi=6550 \mathrm{~m} \cdot \mathrm{s}^{-1}\right)$. The polarization of these

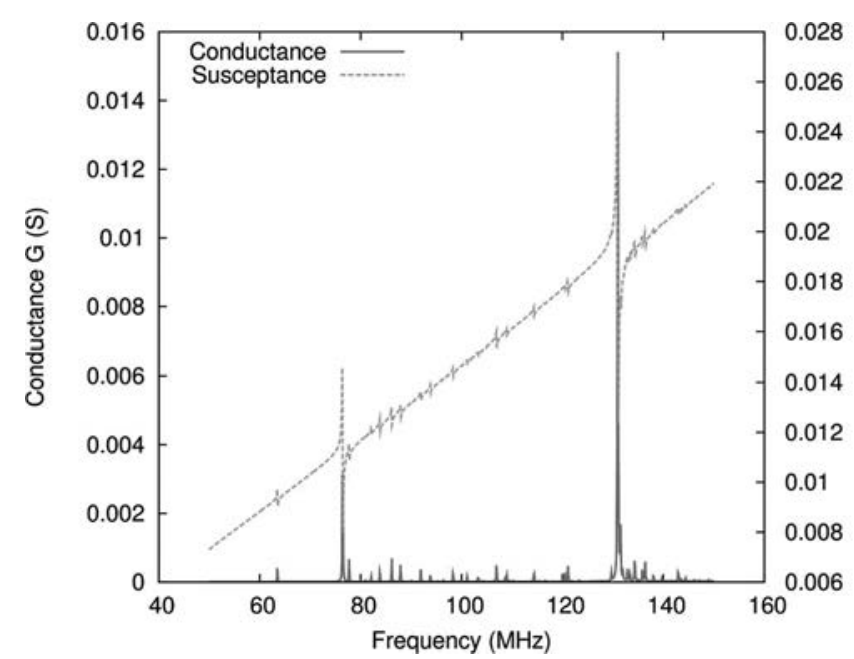

Fig. 3. Theoretical admittance of a $50-\mu \mathrm{m}$-period periodically poled transducer. 
modes has been determined by our FEA simulation tool [7]. The first mode can be compared with the fundamental symmetrical Lamb waves (usually called $\mathrm{S}_{0}$ ). Because the particle motions on each side of the plate exhibit an elliptical shape, this wave is called an elliptic wave hereafter. The second mode is similar to a longitudinal mode with an extension and compression motion of all of the material points of the structure along the propagation direction, and is therefore called a longitudinal wave [9]. In both cases, the resonance occurs at the end of the stopband, although the notion of stopband here is more difficult to comprehend than in the case of SAW operating at Bragg condition. In the later case, the stopband is usually representative of the wave scattering onto the periodic obstacles (electrodes). For PPTs, one can assume that the stopband provides information about dispersion properties of both progressive direct and backward waves forming the mode. Therefore, the width of the frequency stop-band gives an indication of the effectiveness of the mode confinement within the grating.

\section{PPT Fabrication}

A periodically poled transducer is fundamentally based on a periodically poled piezoelectric medium. Such a periodically poled structure can be advantageously achieved on ferroelectric materials such as lead zirconium titanate (PZT) [4], [5] because of the rather small value of its coercive electric field (the absolute value of the electric field above which the spontaneous polarization can be inverted, close to $5 \mathrm{kV} / \mathrm{mm}$ in the case of PZT), or lithium tantalate [10].

As previously presented, all of the test devices have been manufactured using a dedicated poling bench (inspired by [3]) which allows for controlling the poling of optical quality Z-cut $\mathrm{LiNbO}_{3}$ plates. It mainly consists of a high-voltage amplifier used to submit the ferroelectric wafer to an electric field strong enough to invert the negative polarization of the material. The coercive field of lithium niobate is approximately $21 \mathrm{kV} / \mathrm{mm}$ [11], [12]; consequently, the voltage needed to invert the domains is approximately $11 \mathrm{kV}$ for $500-\mu \mathrm{m}$-thick wafers. The domains to be poled are defined using a photo-resist pattern on top of a plate surface, as proposed in [3]. A set of masks has been used for that purpose with poling periods (i.e., acoustic wavelengths) equal to $10,20,40$, and $50 \mu \mathrm{m}$ (corresponding to 5-10-, 20-, and $25-\mu \mathrm{m}$ line widths, respectively). The plate is held in a poly(methyl methacrylate) (PMMA) mounting by means of two O-ring joints which create two cavities filled with a saturated lithium chloride solution used as a liquid electrode (as shown in the scheme of Fig. 4).

An appropriate poling sequence is applied to stabilize the final material state [2], [3], providing reproducible operating conditions and effective poling yields. The poling result can be easily controlled by a simple optical postobservation, because it generates a contrast at the edge of the poled domains.

\section{Experimental Results and Comparison With the Theory}

Each device was found to operate according to theoretical predictions and allowed for electrical measurements. Fig. 5 shows the comparison of experimental and theoretical admittances of a $50-\mu \mathrm{m}$-period PPT.

Although the response is somewhat difficult to interpret because of numerous parasitic bulk wave residuals, two strong contributions are observed at 76 and $130 \mathrm{MHz}$. The first mode corresponds to the elliptic mode and the second mode to the longitudinal mode. It is important to note that the latter cannot be excited with a classical IDT, because the corresponding boundary conditions are difficult to meet with such a transducer. Even if the two modes are slightly shifted considering theoretical predictions, this plot points out the agreement between the results. In a first-order approximation, it is possible to calculate the equivalent phase velocity of each contribution, yielding values of 3800 and $6500 \mathrm{~m} \cdot \mathrm{s}^{-1}$ respectively (in agreement with the predicted values reported in Section II-B). We can, however, observe a difference of magnitude

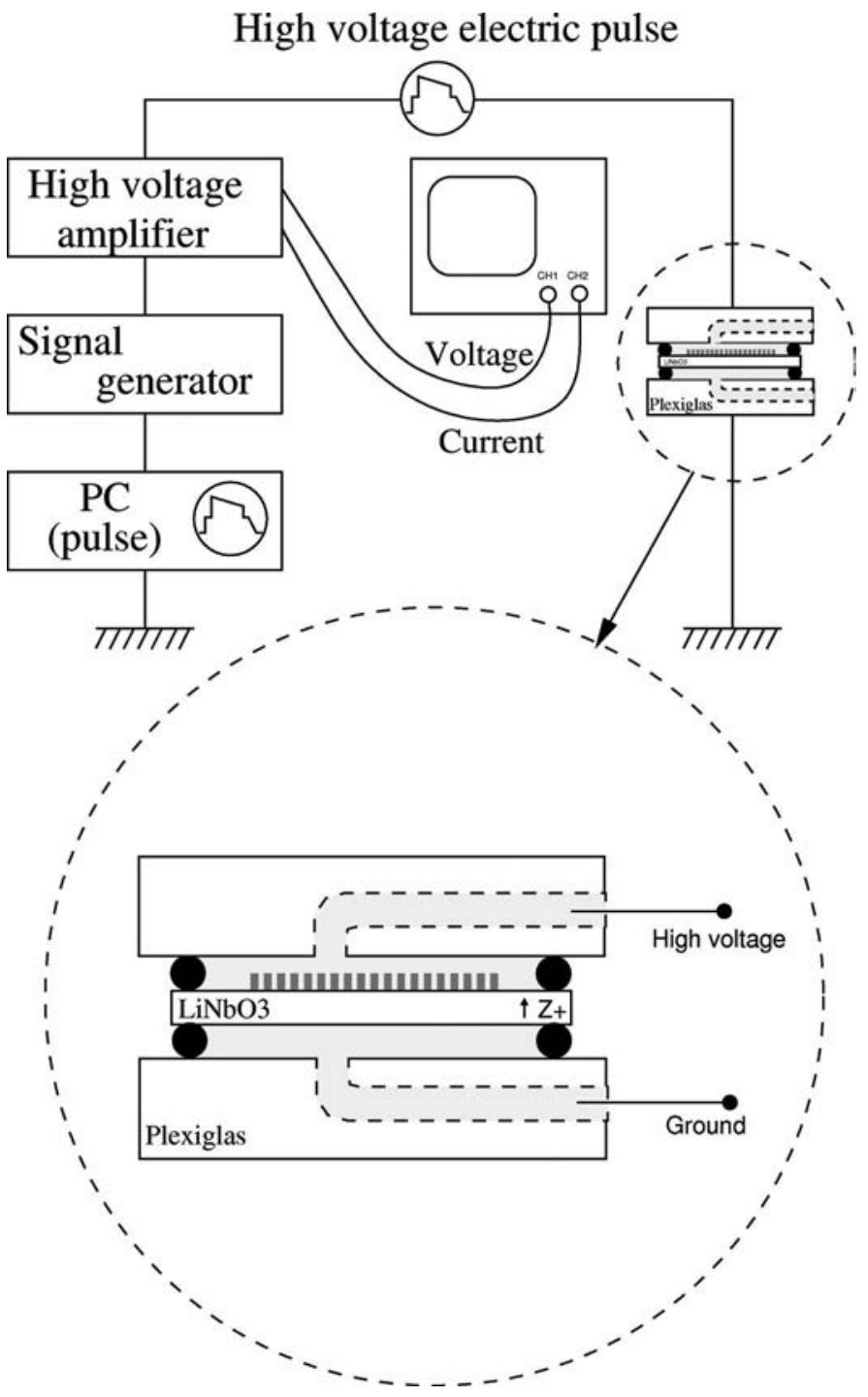

Fig. 4. Schematic diagram of the poling bench. 


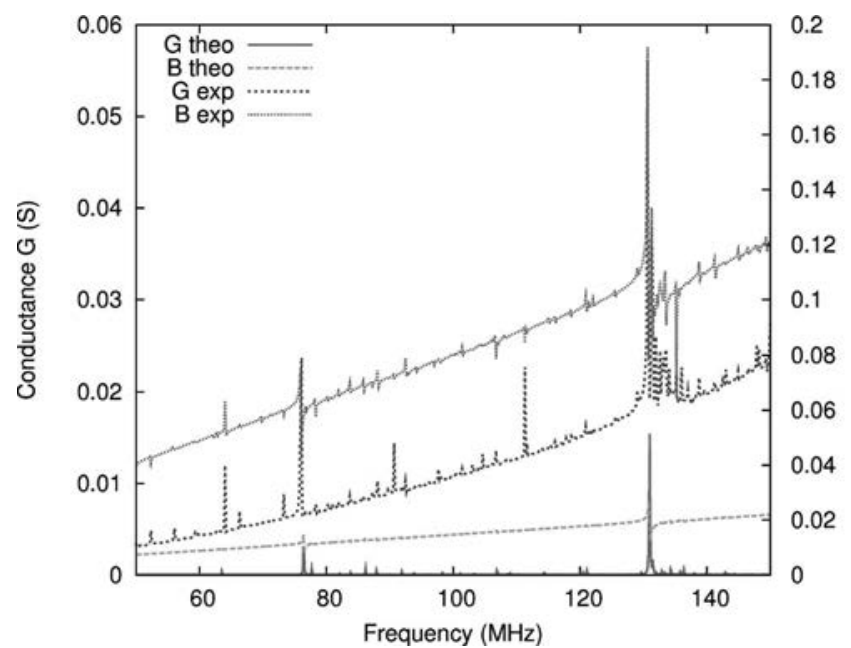

Fig. 5. Comparison between experimental results and theoretical predictions for a $500-\mu \mathrm{m}$-thick $\mathrm{LiNbO}_{3}$ transducer $(\lambda=50 \mu \mathrm{m})$.

between the theoretical and experimental results, which is mainly the result of acoustic losses defined in the simulation tool.

\section{Design of a PPT-Based Filter}

Considering the predictive and manufacturing capabilities, the use of PPTs for the fabrication of band-pass filters in place of IDTs has been investigated. For that purpose, the idea was to attempt to transpose the usual transversal SAW filter techniques [6] to PPTs, taking advantage of the ease of patterning shaped electrodes according to the desired impulse response.

\section{A. Analogy Between IDT-Based Filters and PPT-Based Filters}

Fig. 6(a) presents a classical band-pass IDT-based filter. It consists of two IDTs deposited on a piezoelectric substrate. The transducers are made of interleaved metal electrodes which are used to launch and receive the waves, so that an electrical signal is linearly converted to an acoustic wave and then back to an electrical signal using fundamental piezoelectric properties. A basic advantage is that acoustic waves travel very slowly (typically $3000 \mathrm{~m} \cdot \mathrm{s}^{-1}$ ), so large delays can be obtained with smaller device dimensions compared with electromagnetic delay lines. In designing a SAW filter, the overall frequency response characteristics are determined by deriving two impulse responses for the two transducers, whose transforms are added together in decibels [13].

In this configuration, a rectangular frequency response should be obtained. Therefore, this concept has been used to design our PPT-based filters [see Fig. 6(b)] assuming that the waves emitted by PPTs actually are propagating like IDT-excited waves.

In our PPT-based filters, the electrode shapes are designed on previously poled substrates according to the desired frequency operation. To validate the principle, it was first decided to design basic electrode shapes using rectangles and diamonds. In theory, with those transducers, the frequency responses should be obtained as:

- $(\sin X / X)^{2}$ for a filter based on 2 rectangular electrodes,

- $(\sin X / X)^{3}$ for a filter based on one rectangular electrode and one diamond-shaped electrode,

- $(\sin X / X)^{4}$ for a filter based on 2 diamond-shaped electrodes.

The frequency response for those 3 different filters is presented in Fig. 7. Although usually geometric tapering of both IDTs is not preferred using standard SAWs (one is geometrically tapered and the second one is functionalized using source suppression), all of these configurations were tested to exemplify the actual operation and interaction of PPTs.

\section{B. Fabrication of the PPT-Based Filters}

The first step of the fabrication consists of poling the piezoelectric substrate; in our case, a 3 -in $(76.2-\mathrm{mm}) \mathrm{LiN}$ $\mathrm{bO}_{3}$ wafer. A $40-\mu \mathrm{m}$ poling period was used, corresponding to the excitation of the elliptically polarized wave near $100 \mathrm{MHz}$. Several electrodes of various shapes and lengths were then patterned on the different poled areas to test many configurations. Several different gaps between the input and output electrodes have also been studied. The chosen design is shown in Fig. 8.
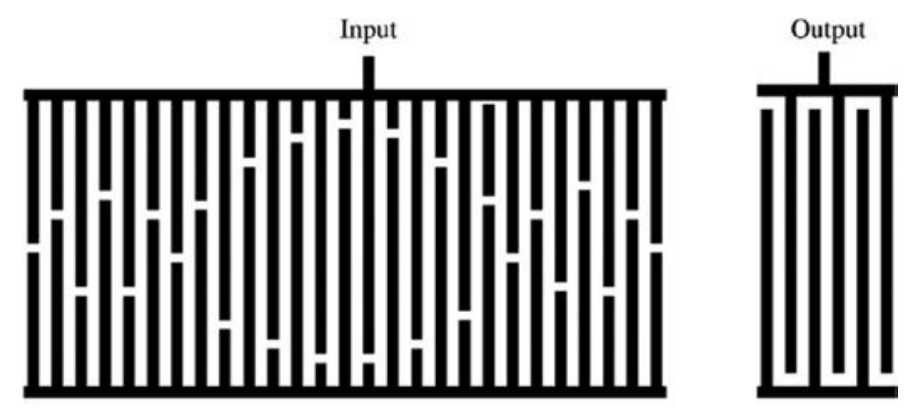

(a)

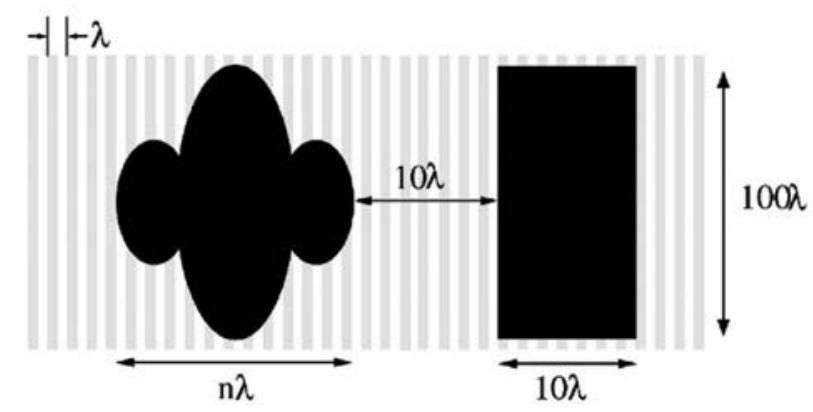

(b)

Fig. 6. Comparison between interdigital transducer-based and periodically poled transducer-based band-pass filters. 


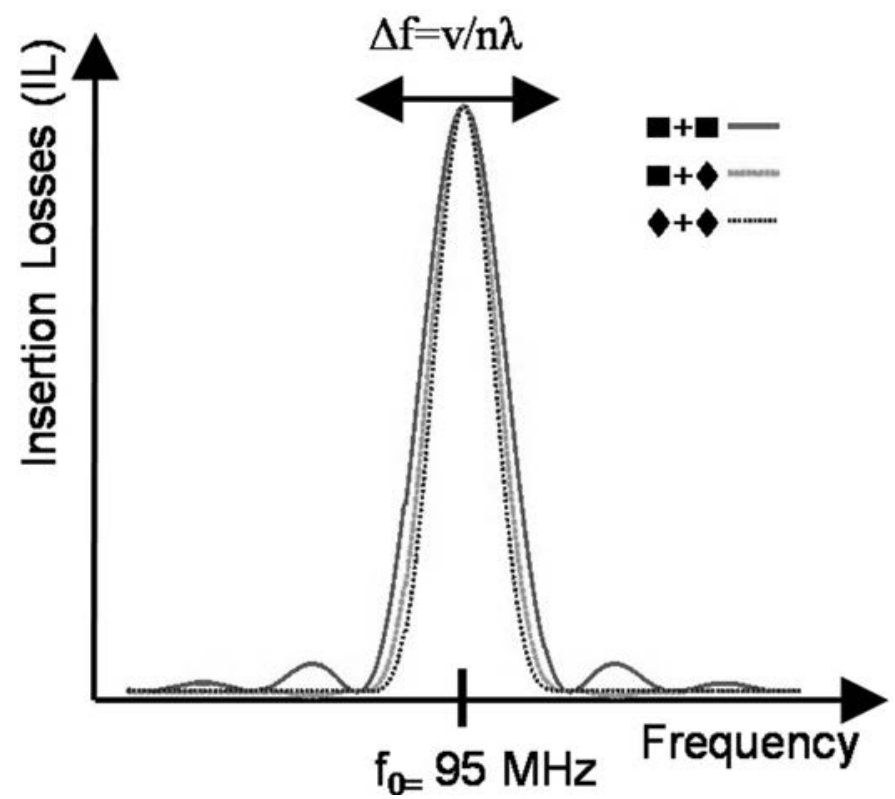

Fig. 7. Frequency responses which should be obtained with three different periodically poled transducer-based filters.

Finally, the wafer is diced so that each poled area has access to enough electrodes on each device (typically 2 to 4) for testing purposes.

\section{EXPERIMENTAL RESUlts}

\section{A. Acoustic Measurements}

Transmission measurements have been performed by means of a tip-probing bench linked to a ZVC network analyzer (Rohde \& Schwarz, Munich, Germany). Although all the transducers exhibited reflection responses in agreement with theoretical predictions, only one device was found to present a significant transfer function according
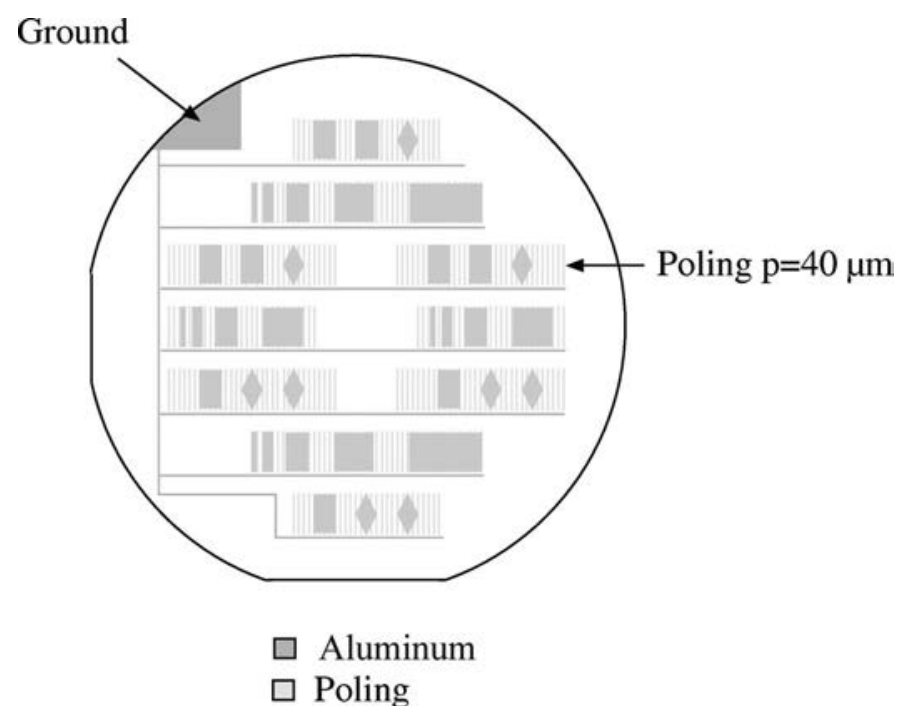

Fig. 8. Several designs used to validate the periodically poled transducerbased filter principle.

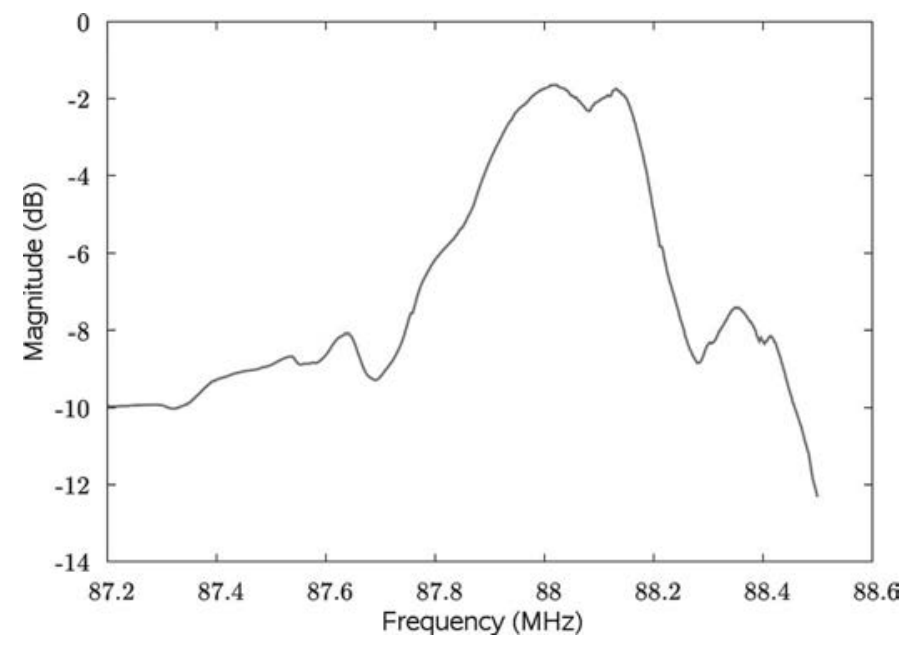

Fig. 9. Transfer function obtained with a filter composed of two rectangular electrodes ( $10 \lambda$ and $20 \lambda$, respectively) separated by $15 \lambda$ : typical signature of a band-pass filter.

to the expected filtering function. This device was composed of two rectangular electrodes whose characteristics are:

- First rectangle electrode length: $10 \lambda$

- Gap between the electrodes: $15 \lambda$

- Second rectangle electrode length: $20 \lambda$

As mentioned in the preceding sections, this device exhibited a multi-mode response because of the $\mathrm{LiNbO}_{3}$ thickness. However, two contributions are easily observed at around 88 and $95 \mathrm{MHz}$. When zooming-in near the first one, the typical signature of a band-pass filter can be observed (see Fig. 9).

The characteristics of this filter are: a center frequency of $88 \mathrm{MHz}$, a passband width equal to $350 \mathrm{kHz}$ and insertion losses of about 1.6 dB. Furthermore, two peaks can be clearly observed on the top of the passband width. It seems that two modes contribute to the response, which suggests that the filter is based on a coupling mode mechanism.

To validate this hypothesis, the reflection coefficient, S11, of one of the transducers was measured; see Fig. 10.

This plot actually shows two principal reflection contributions coinciding with the previously mentioned peaks of the transfer function. Because the distance between the two transducers is relatively small (only $15 \lambda$ ), the possibility of coupling energy from another transducer via evanescent wave behavior is realistic. This reinforces the interpretation of the stopband of the transducer dispersion curve even in the second-harmonic regime (stationary wave conditions). Furthermore, it would explain the operation of the filter reported in [10] on periodically poled lithium tantalite, which exhibits a behavior very close to the one described here. To better understand the transducer operation, optical measurements have been performed to observe the acoustic vibration distribution under and near the PPT. One can also observe a resonance with extremely low insertion losses (about $0.3 \mathrm{~dB}$ ) 


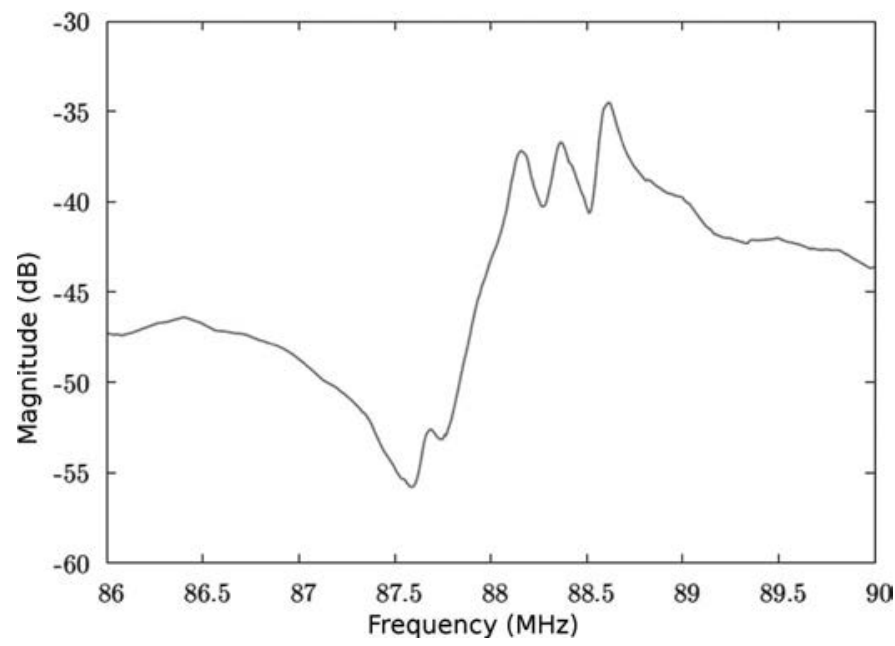

Fig. 10. Reflection coefficient S11 observed for one of the two transducers of the operating filter.

at $95 \mathrm{MHz}$ (see Fig. 11). Although not well explained, this result is very encouraging because such a resonator could be advantageously used to fabricate an oscillator because the unloaded quality factor was revealed to be close to 50000 at about $100 \mathrm{MHz}$.

To better understand the transducer operation and the way the filter works, acoustic-optical measurements were performed to observe the acoustic vibration distribution under and near the PPT.

\section{B. Acoustic-Optical Measurements}

As previously explained, acoustic-optical measurements were implemented here to analyze the mechanical wave distribution during the electrical excitation of an electrode. The experimental setup used for the measurements of the elastic wave displacement is a scanning optical heterodyne interferometer similar to the one described in [14], but here only allowing amplitude detection. The probe basically consists of a Michelson interferometer folded into a single arm, in which the output coupler of the laser is used

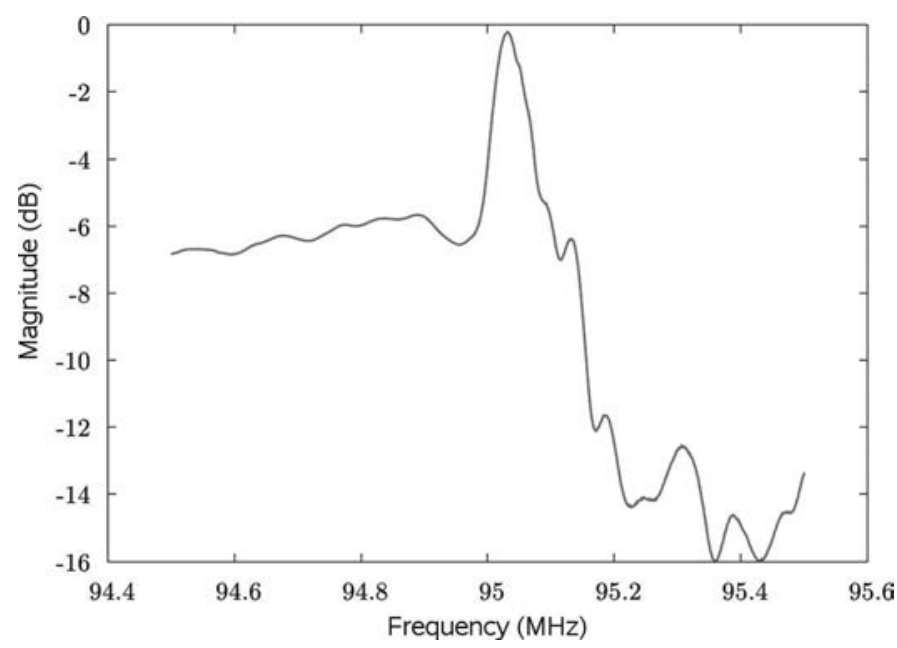

Fig. 11. Resonance observed at $95 \mathrm{MHz}$ on the same device.

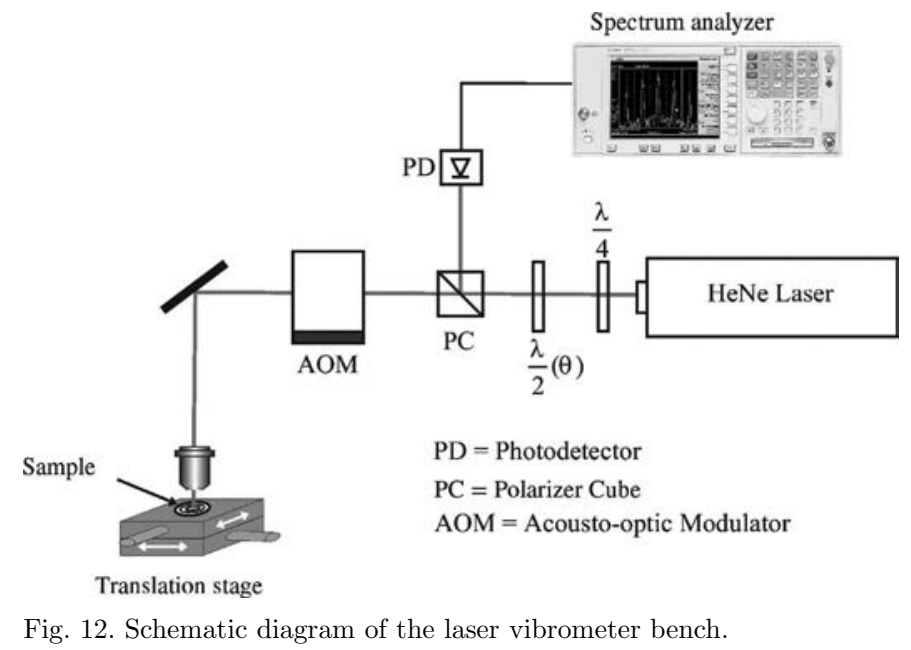

as one of the mirrors constituting the interferometer. A schematic of this heterodyne laser vibrometer is displayed in Fig. 12.

The 500- $\mu \mathrm{m}$-thick $40-\mu \mathrm{m}$-period transducer was then characterized using this experimental protocol. The upper electrode corresponds to a few tens of wavelengths metal layer deposited on a several-millimeter-long periodically poled area.

Only modes having a significant deformation along the axis normal to the plate (here the $\mathrm{Z}$-axis) are detectable by the laser vibrometer. Therefore, the distribution of the elliptic mode propagation at $95 \mathrm{MHz}$ was particularly observable during this experiment. Fig. 13 shows the amplitude variation of the material vibrations around the excited electrode.

A maximum of vibration amplitude of about $0.2 \mathrm{~nm}$ can be observed with a waviness actually corresponding to the grating periodicity. Moreover, one can observe that the vibrations are mainly localized under the electrode, although some vibration traces are recorded beside the excited surface.

For an in-depth analysis of this phenomenon, a graph representing a cross-section of the previous figure along the y-axis has been plotted (see Fig. 14).

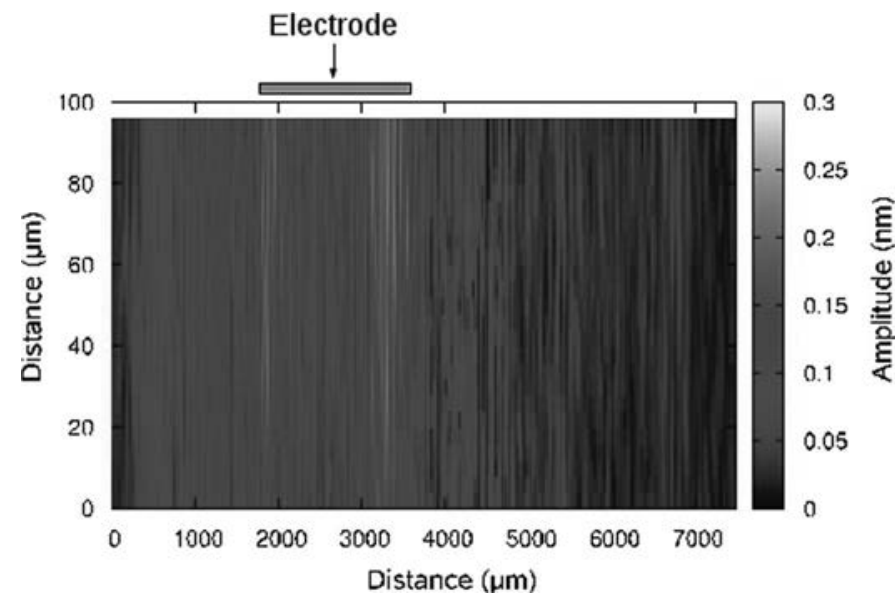

Fig. 13. Acousto-optical scan performed using a laser vibrometer showing the amplitude variation of the vibrations versus the distance. 


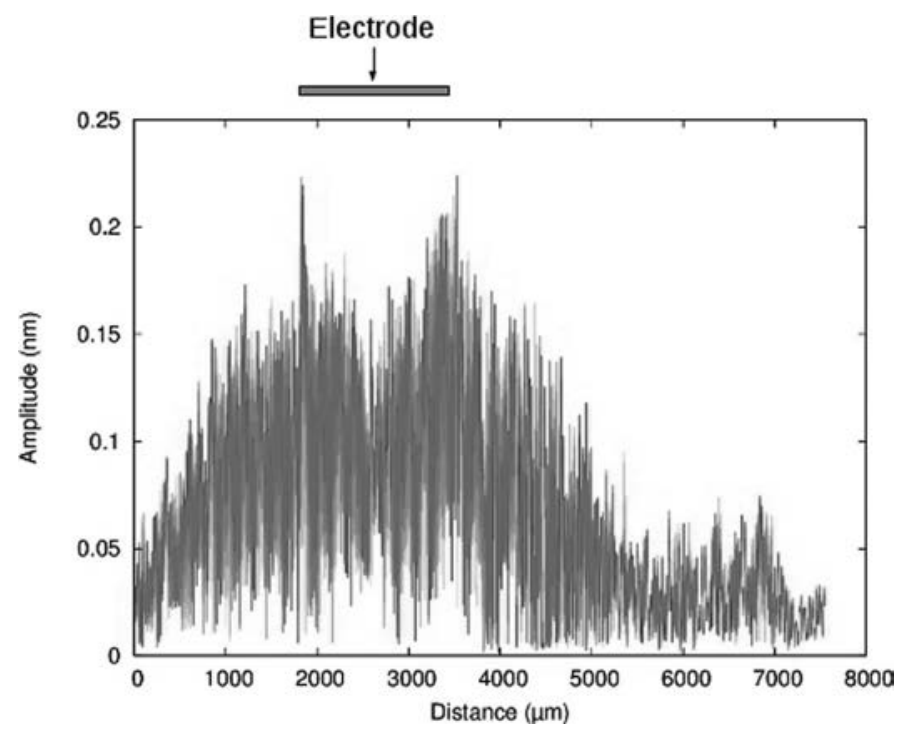

Fig. 14. Section view along the $y$-axis of Fig. 13, showing the amplitude of material vibrations versus the distance.

This graph confirms that the maximum of vibration amplitude is located under the electrode. These vibrations exponentially decrease over a distance of $1 \mathrm{~mm}$ on both sides of the electrode. This measurement confirms that the elastic waves excited by a PPT are quasi-standing waves which extend only a few wavelengths outside the electrode regions. Finally, these observations are in agreement with the operation principle reported in Section I-B. The excitation of the grating is achieved for a second-harmonic operation condition of the grating, yielding the superimposition of progressive direct and backward waves, which does not allow for launching waves in a manner similar to IDTs. This result explains why the only operating PPTbased filter is the one whose electrodes are the closest, allowing for a mode-coupling operation of the resulting quadripole.

The same experiment was performed with an electrode with a different shape (i.e., a diamond). The same behavior as the one reported here was observed, i.e., the localization of the principal surface motion right under the electrodes and an evanescent regime outside the metalized area. These results indicate that the propagation of elastic waves in a PPT does not depend on the electrode shape. This result is crucial because it demonstrates that the initially proposed approach is not well adapted for the fabrication of transversal filters by simply transposing classical IDT concepts to PPT. However, it also provides essential information about PPTs operation and the possibility for coupling modes from one transducer another. The very small losses observed on the corresponding transfer function also suggest that such transducers should be particularly well adapted for such filter operations.

\section{CONCLUSION}

In this paper, a new concept of acoustic band-pass filters has been presented. This device is based on the use of PPTs in lithium niobate instead of classical IDTs. The possibility of fabricating PPTs in lithium niobate has been demonstrated for different periods. This kind of transducer enables excitation of an elliptical mode with a resonance frequency twice as high as a wave excited by a classical IDT with the same mechanical period. This device is thus very interesting for the fabrication of RF acoustic devices such as band-pass filters.

For that purpose, PPT-based filters were fabricated, copying the principle of IDT-based transversal filters. Although most of the tested structures did not exhibit the expected transfer functions, one device did present a transfer function with marked rectangular spectral windows close to the expected response. The analysis of the electrical parameters of the device has oriented the investigation toward a coupled-mode operation regime. Acoustic-optical measurements have indirectly confirmed this hypothesis. It appears that the elastic waves excited by a PPT are quasi-standing waves, with electrically stimulated vibration clearly localized under the metalized area and an evanescent behavior in the adjacent grating. As a consequence, even if simple transversal filter operations are still beyond reach because of the inner transducer principle, the possibility of fabricating coupled-mode filters is clearly emphasized and exemplified here, with remarkable loss features and compactness characteristics related to the PPTs' fundamental properties. Although more work is clearly needed to set definitive design rules for such transducer, the path is open for an effective alternative to SAW for RF filter manufacturing exploiting periodically poled structures.

\section{REFERENCES}

[1] C. C. W. Rüppel and T. A. Fjeldly, Eds. Advances in Surface Acoustic Wave Technology, Systems And Applications, vol. 1 and 2, Singapore: World Scientific, 2001.

[2] E. Courjon, N. Courjal, W. Daniau, G. Lengaigne, L. GauthierManuel, and S. Ballandras, "Lamb wave transducer built on periodically poled Z-cut $\mathrm{LiNbO}_{3}$ wafers," J. Appl. Phys., vol. 102, no. 11, art. no. 114107, 2007.

[3] L. E. Myers, R. C. Eckardt, M. M. Fejer, R. L. Byer, W. R. Bosenberg, and J. W. Pierce, "Quasi-phase-matched optical parametric oscillator," J. Opt. Soc. Am. B, vol. 12, no. 11, pp. 2102-2116, 1995.

[4] S. Ballandras, W. Daniau, B. Gautier, D. Hauden, M. Wilm, V. Laude, V. Ruch, and S. Flambart, "A novel surface wave transducer based on periodically poled piezoelectric domain," in Proc. Joint European Frequency and Time Forum-IEEE Int. Frequency Control Symp., 2003, pp. 893-896.

[5] A. K. Sarin Kumar, P. Paruch, J.-M. Triscone, W. Daniau, S. Ballandras, L. Pellegrino, D. Marré, and T. Tybell, "A high frequency surface acoustic wave device based on thin film piezoelectric interdigital transducers," Appl. Phys. Lett., vol. 85, no. 10, p. 1757-1759, 2004.

[6] D. P. Morgan, Wave Devices for Signal Processing, 2nd ed. (Studies in Electrical Eng. 19). Amsterdam, The Netherlands: Elsevier, 2007.

[7] S. Ballandras, M. Wilm, P.-F. Edoa, A. Soufyane, V. Laude, W. Steichen, and R. Lardat, "Finite-element analysis of periodic piezoelectric transducers," J. Appl. Phys., vol. 93, no. 1, pp. 702-711, 2003.

[8] IEEE Standard on Piezoelectricity. IEEE Std. 176-1949, 1949.

[9] E. Courjon, J. Hauden, J. Masson, D. Gachon, L. Gauthier Manuel, W. Daniau, N. Bodin, and S. Ballandras, "Pure longitudinal plate mode excited by poled domains transducers on $\mathrm{LiNbO}_{3}$," in Proc. IEEE Int. Frequency Control Symp., 2007, pp. 1073-1076. 
[10] M. Kadota, T. Ogami, K. Yamamoto, and Y. Cho, "Acoustic wave devices using periodical poled Z-cut $\mathrm{LiTaO}_{3}$ plate," in Proc. Joint IEEE Int. Frequency Control Symp.-European Frequency and Time Forum, 2009, pp. 919-922.

[11] M. C. Wengler, M. Müller, E. Soergel, and K. Buse, "Poling dynamics of lithium niobate crystals," Appl. Phys. B, vol. 76, no. 4, pp. 393-396, 2003.

[12] S. Kim, V. Gopalan, and A. Gruverman, "Coercive fields in ferroelectrics : A case study in lithium niobate and lithium tantalate," Appl. Phys. Lett., vol. 80, no. 15, pp. 2740-2742, 2002.
[13] D. Royer and E. Dieulesaint, Ondes Élastiques Dans les Solides, Tome 2: Génération, Interactions Acousto-Optiques, Applications. Paris, France: Masson, 1999.

[14] P. Vairac and B. Cretin, "New structures for heterodyne interferometric probes using double-pass," Opt. Commun., vol. 132, no. 1-2, pp. 19-23, 1996. 